\title{
Voluntary Chronic Exercise Decreases Nociceptive Responses Before and After a Traumatic Stressor
}

\author{
Sarah Ford Mozingo, M.A. \\ Department of Psychology, Southeastern Louisiana University, Hammond, LA, United \\ States of America
}

Brandon A. Baiamonte, Ph.D. (Corresponding Author)

Department of Psychology, Southeastern Louisiana University, Hammond, LA, United States of America

SLU Box 10831, Hammond, LA 70402

Tel: 985-549-2154 Fax: 985-549-3892 Email: brandon.baiamonte@ selu.edu

Daniel B. Hollander, Ed.D.

Department of Kinesiology and Health Studies, Southeastern Louisiana University, Hammond, LA, United States of America

Tulane University, School of Professional Advancement, New Orleans, LA, United States of America

Robert R. Kraemer, Ed.D.

Department of Psychology, Southeastern Louisiana University, Hammond, LA, United States of America

The authors have no competing interests in relation to the work described in this manuscript.

Received: September 3, 2019

doi:10.5296/jbls.v11i1.15396
Accepted: October 16, 2019

URL: https://doi.org/10.5296/jbls.v11i1.15396 


\section{Abstract}

Objective: The hypoalgesic effect of acute bouts of exercise on healthy participants has been well documented across a variety of exercise modalities. However, the effects of a long-term exercise regimen on nociception is fairly unknown. Furthermore, it is unclear if exercise can be an effective treatment for disorders such as posttraumatic stress disorder, which can be characterized by hyperalgesia. The purpose of the current study is to investigate the effects of chronic exercise on thermal nociception before and after a traumatic stressor.

Methods: Twenty-four C57/B6 mice were separated into two groups with 12 animals performing voluntary wheel running over a 5 week period. All mice were assessed weekly to determine the effects of chronic exercise or rest on thermal nociception. After 4 weeks, animals were further divided into 4 groups according to exercise and traumatic stress exposure. All mice were exposed to a 15 minute predator odor paradigm with "no stress" animals being exposed to ambient air and "stress" animals exposed to bobcat urine. All mice were tested 48 hours post exposure for changes in thermal nociception.

Results: Findings from this study yield support that chronic, voluntary exercise decreases thermal nociception in mice over a 4-week period before a traumatic stressor and 48 hours after exposure to the stressor.

Conclusion: In conclusion, voluntary, long-term exercise has been shown to elevate nociceptive thresholds, providing support for these exercise programs in the treatment of stress-induced hyperalgesia.

Keywords: stress, exercise, nociception, PTSD, pain

\section{Introduction}

Approximately 223.4 million Americans have experienced a traumatic event, such as a natural disaster, terrorist attack, rape, or death, in their lifetime. Of this subset, 20\% (approximately 44.7 million) go on to develop and struggle with posttraumatic stress disorder (PTSD) (PTSD United, 2013). PTSD can develop in adults or children and may lead to intrusive recollection of the trauma (i.e. flashbacks or nightmares), avoidance of trauma-related stimuli, and hyperarousal, such as irritability, heightened startle reaction, and pain sensitivity (hyperalgesia) (American Psychiatric Association [APA], 2013; US Department of Veteran Affairs, 2017; Whitaker, Farooq, Edwards \& Gilpin, 2016). The prevalence of PTSD and the highly associated economic and personal costs necessitate that healthcare providers develop more effective and conservative treatments for military personnel and civilians suffering from this disorder. Exercise is a therapy often utilized to treat a multitude of disorders, including PTSD and pain sensitivity. However, the influence of chronic exercise on nociception is not fully understood. Therefore, the purpose of this study was to examine the effects of long-term, chronic exercise on nociception before and following a traumatic stressor.

Individuals with PTSD frequently demonstrate co-occurring pain issues as well as those suffering from chronic pain disorders often present PTSD symptoms (Asmundson, Coons, 
Taylor \& Katz, 2002). Furthermore, previous research suggests that the co-occurrence of PTSD and chronic pain exacerbate the negative effects of each other, leading to poorer outcomes and treatment complication (Asmundson \& Katz, 2008). Supporting this relationship, Defrin and colleagues (2008) found that participants with PTSD demonstrate greater comorbid chronic pain symptomology than those with anxiety disorders or healthy controls. Further support of PTSD association with pain hypersensitivity was from a study reporting higher pain sensitivities from cold pressor task and overall poorer psychiatric and physical functioning in 61 participants with PTSD compared to 33 participants with anxiety disorders and controls (Mostoufi et al., 2014). Despite the growing body of research establishing the link between these two disorders, the mechanism behind pain and PTSD comorbidity is largely misunderstood (Asmundson \& Katz, 2008). However, one of the most recently investigated mechanisms of interest, points to elevations in corticotropin-releasing factor (CRF) levels in the central amygdala (CeA) following a traumatic stressor in an animal model (Itoga et al., 2016). Not only did stressed animals demonstrate anxiety-like behaviors in the conditioned-place aversion protocol, they also exhibited significantly reduced nociceptive thresholds (hyperalgesia or pain-sensitivity) compared to controls. These changes induced by a predator odor were attenuated by systemic and central amygdala (CeA) administration of a $\mathrm{CRF}_{1}$ receptor antagonist. These findings along with additional research, suggest that $\mathrm{CRF}$ signaling in the $\mathrm{CeA}$ may be responsible for thermal hyperalgesia (pain-sensitivity) demonstrated in rats following a traumatic stressor (Defrin et al., 2008; Itoga et al., 2016; Adamec, Fougere \& Risbrough, 2010; Roltsch et al., 2014).

\subsection{Exercise and Pain Perception}

Exercise has also been shown to alter pain perception in human and animal models and is frequently implemented in treatment programs for disorders like PTSD and chronic pain (APA, 2013; Kotlyn, 2000; Kotlyn \& Arbogast, 1998). Kotlyn and Arbogast (1998) found evidence for resistance exercise altering pain processing in humans as demonstrated by exercising individuals reporting higher pain thresholds five minutes after exercise than those who were in a quiet rest condition. Baiamonte and colleagues (2016) further explored the impacts of exercise-induced hypoalgesia (EIH) following dynamic circuit resistance exercise and results indicated pain tolerance significantly increased following a resistance exercise circuit, but no significant changes were demonstrated for pain threshold, further illustrating exercise's impact on pain processing. Moreover, a meta-analysis performed by Naugle, Fillingim, and Riley (2012) examined twenty-five different studies that explored the relationships between different exercise types (aerobic and isometric and dynamic exercises) and hypoalgesia. Results indicated a reduction in pain perception following all three forms of exercise, as well as in many different forms of pain assessment (Naugle et al., 2012). These findings involving hypoalgesia and exercise may prove exercise to be an effective method to alter pain perception in healthy participants as well as patients with chronic pain. Furthermore, the hypoalgesic effect of exercise may be useful in the treatment of PTSD symptomology such as hyperalgesia. However, it must be noted that the hypoalgesic effect of acute exercise was transient with pain measures returning to pre-exercise baseline scores within fifteen minutes. In addition, there is a paucity of studies measuring the hypoalgesic effect of 
long-term, chronic exercise, with most studies only investigating pain perception following one acute bout of exercise. An investigation of nociceptive changes after multiple bouts of exercise is needed to fully understand how exercise alters pain processing.

\subsection{Exercise as an Intervention for PTSD}

Empirical evidence has emerged supporting exercise as a promising intervention for mental and physical health in numerous studies (Fetzner \& Asmundson, 2015; Powers et al., 2015). Furthermore, exercise augmented exposure therapy for PTSD has been shown to improve symptoms and increase brain-derived neurotropic factor (BNDF), which modulates synaptic plasticity of the brain and has been associated with improved cognitive functions and abilities, such as learning and memory (Hides, Jull \& Richardson, 2001). Cortisol responses to a dexamethasone suppression test in resting, endurance-trained participants are similar to responses from untrained participants (Duclos, Gouarne \& Bonnemaison, 2016; Gouarne, Groussard, Gratas-Delamarche, Delamarche \& Duclos, 2005). Moreover, endurance-trained athletes have similar resting HPA activity as untrained healthy men and exercise training does not induce hypercortisolism. However, when HPA axis activity is stimulated repeatedly, trained athletes reveal reduced tissue sensitivity to stimulation from elevated glucocorticoids (Duclos et al., 2016). Taken together, these findings not only implicate exercise's ability to reduce PTSD-induced anxiety and cognitive symptoms, but also demonstrated exercise to alter glucocorticoids, which are reduced in individuals with PTSD (Duclos et al. 2016; Droste et al., 2007).

\subsection{Specific Aims}

As previously stated, increased $\mathrm{CRF}$ has been demonstrated following traumatic stress models in animals and plays a role in pain processing (Adamec et al. 2010; Edwards et al., 2012; Ji \& Neugebauer, 2007; Ji \& Neugebauer, 2008). More specifically, past findings have indicated that greater levels of CRF are associated with increased PTSD-like symptoms and higher nociception (pain-sensitivity) (Itoga et al., 2016; Roltsch et al., 2014). However, chronic exercise has demonstrated reductions in PTSD symptoms in animals and humans and may attenuate nociceptive changes in post-stress models. Moreover, chronic exercise has been shown to reduce adrenocorticotrophic hormone (ACTH) responses to infusion of CRF, (Watanabe, Morimoto, Sakata, Wada \& Murakami, 1991) a peptide with extensive links to both nociception and anxiety-like symptoms. Therefore, chronic exercise may be an effective intervention for reducing hyperalgesia commonly experienced in PTSD. The purpose of the study was to examine the effects of chronic voluntary exercise via running wheel on thermal nociception over a 4-week period. In addition, we examined the effects of chronic exercise on nociception following exposure to predator odor stress to further understand the link between nociception, exercise, and traumatic stress.

\section{Method}

\subsection{Animals}

Twenty-four male and female C57/ B6 mice were bred from an existing colony on Southeastern Louisiana University campus and all mice were weaned at 6 weeks. Prior to the 
start of experiments, mice were single housed in a humidity and temperature-controlled $\left(22{ }^{\circ} \mathrm{C}\right.$ ) vivarium on a reverse 12-h light/dark cycle (lights off at 9 a.m.). Food and water was available ad libitum and all behavioral tests were conducted during the dark period. All mice were acclimated to the experiment room for 1 week before start of experiments. Acclimation consisted of placing animals in the testing room for 15 minutes each day for 1 week to allow for exploration. All animal experiments were approved by Southeastern Louisiana University's Institutional Animal Care and Use Committee (IACUC) in accordance with the National Institute of Health guidelines.

\subsection{Hargreaves Method}

To assess thermal nociception, each hindpaw was stimulated on the plantar surface using a halogen heat source from an IITC model 309 Hargreaves apparatus (IITC Life Science, Inc., Woodland Hills, CA). The Hargreaves apparatus consisted of Plexiglass enclosures on glass floors suspended $30 \mathrm{~cm}$ from tabletop. Each mouse was placed in the enclosure and latency to withdraw the stimulated paw was assessed via a digital display in seconds. The intensity of the heat source was set at 50 A.I. to establish a baseline withdrawal latency of approximately 8-10 seconds. A 30-s cut-off was employed to prevent tissue damage in non-responsive subjects. On test days, mice were placed in the examination room for 5 minutes to allow adjustment to the light and testing environment, followed by another 5 minutes in the testing apparatus for acclimation as well. On each test day, each hind paw was tested twice in alternating order (i.e. left, right, left, right), allowing for a 1 minute break in between both sets of stimulations. These scores were averaged to yield a single score for analysis. This average hindpaw withdrawal latency was the thermal nociception score for each mouse (i.e. lower scores indicate hyperalgesia).

\subsection{Voluntary Exercise Paradigm}

After 1 week in which pre-exercise baselines were determined, the experimental groups, "exercise control" and "exercise with predator odor stressor", were transferred to home cages with running wheels $(25 \mathrm{~cm}$ in diameter). The wheels were part of the cage and animals were free to enter or exit the wheel at their own leisure. Wheel cycle rotations were measured via a digital wheel-turning count system and recorded every 24-hours during week days and 48-hours for weekends before the dark phase of the diurnal cycle. Housing conditions for "non-exercising with predator stressor" and "controls" were unchanged. Mice exercised for 4 weeks before exposure to predator odor.

\subsection{Stress Exposure}

Following 4 weeks of exercise, mice were transferred from home cage to a clean cage and then exposed to predator odor (bobcat urine) or ambient air for 15 minutes. Urine was added to a sponge that was placed inside the cage in which the mice had direct contact with the urine and sponge. This model of traumatic stress has been shown to produce avoidant behavior, anxiety-like behaviors, and hyperalgesia (Roltsch et al., 2014). Mice were then transferred back from experimental cages to home cages. 


\section{Experimental Procedures}

\subsection{Experiment 1}

Hargreaves testing over time. Mice were tested for baseline thermal nociception every day for 1 week using the Hargreaves test until nociceptive responses stabilized. Then animals were counterbalanced into 1 of 2 groups (non-exercise vs. exercise). All animals were tested once a week over 4 weeks to determine whether chronic exercise altered thermal nociception.

\subsection{Experiment 2}

stress and exercise effects on thermal nociception. Following 4 weeks of exercise and nociceptive testing, animals were further divided into 1 of 4 groups (non-exercise/neutral; non-exercise/stress; exercise/neutral; exercise/stress). All mice went through the 15-minute predator odor paradigm with neutral mice being exposed to ambient air and stress mice being exposed to bobcat urine. All mice were tested 2 days following exposure for any changes in thermal nociception. It must be noted that the exercising animals were still allowed to exercise following exposure to either bobcat urine or ambient air.

\section{Statistical Analysis and Results}

All data were analyzed using IBM Statistical Package for the Social Sciences (SPSS) version 25 and statistical significance was set at $p<0.05$. Each testing day, the 4 withdrawal latencies (thermal nociception) were averaged into one score for each subject. To determine the effects of chronic exercise on nociception, a mixed-factorial analysis of variance (ANOVA) was conducted using time as the within subjects factor (BL vs. week 1 vs. week 2 vs. week 3 vs. week 4) and condition as the between subjects variable (non-exercising vs. exercising). All significant main effects were analyzed using pairwise comparisons and T-tests with Bonferroni corrections were conducted to determine whether a significant interaction occurred.

To determine the effects of traumatic stress on nociception and whether chronic exercise alters these changes, a $(2 \times 2 \times 2)$ factorial ANOVA with interactions of exercise (exercise vs. non-exercise), posttraumatic stress (stress vs. non-stress), and time (pre-stress baseline vs. $48 \mathrm{hr}$. post-stress) on thermal nociception were analyzed. The 4 scores of nociception over the 4-week period of exercise were averaged to produce 1 score for each animal. This served as the "pre-stress" baseline. All significant main effects were analyzed using pairwise comparisons and post-hoc analyses utilizing T-tests with Bonferroni corrections were conducted to determine whether a significant interaction occurred.

\section{Results}

\subsection{Chronic Exercise on Nociception}

To analyze the effects of chronic exercise on thermal nociception (as indicated by withdrawal latencies), a mixed-factorial ANOVA was conducted with time as the within-subjects factor (BL vs. week 1 vs. week 2 vs. week 3, vs. week 4) and condition as the between subjects (non-exercising vs. exercising). Means and standard deviations are reported in Table 1. 


\section{Macrothink}

Journal of Biology and Life Science

ISSN 2157-6076 2020, Vol. 11, No. 1

Animals in exercising and non-exercising conditions did not statistically differ in withdrawal latencies at baseline, $p>.05$. There was a significant main effect for exercise condition on thermal nociception, $F(1,22)=7.49, p=0.01$, indicating exercising animals displayed higher thermal withdrawal latencies than non-exercising animals. Additionally, there was a significant interaction effect between time and the exercise condition used, $F(4,88)=4.53, p$ $=0.002$. Post-hoc analyses of T-tests with Bonferroni corrections were conducted on the interaction and indicate significantly higher withdrawal latencies for exercising mice compared to non-exercising mice at week 3 of data collection $(p=0.007)$ and at week $4(p=$ 0.008). There was not a significant main effect for time on thermal nociception. Data are summarized in Figure 1.

Table 1. The means and standard deviations for exercise condition on withdrawal latencies over time

\begin{tabular}{lcccccccc}
\hline & \multicolumn{3}{c}{ Exercising Mice } & & \multicolumn{3}{c}{ Non-exercising mice } \\
Withdrawal latencies & $M$ & $S D$ & $n$ & & $S D$ & $n$ \\
\hline Hargreaves baseline & 9.45 & 1.83 & 12 & 10.71 & 2.28 & 12 \\
Hargreaves week 1 & 13.63 & 5.57 & 12 & 10.28 & 2.80 & 12 \\
Hargreaves week 2 & 11.83 & 6.31 & 12 & 8.31 & 2.37 & 12 \\
Hargreaves week 3 & 12.83 & 4.84 & 12 & 8.20 & 2.37 & 12 \\
Hargreaves week 4 & 15.39 & 7.86 & 12 & 8.54 & 2.27 & 12 \\
\hline
\end{tabular}



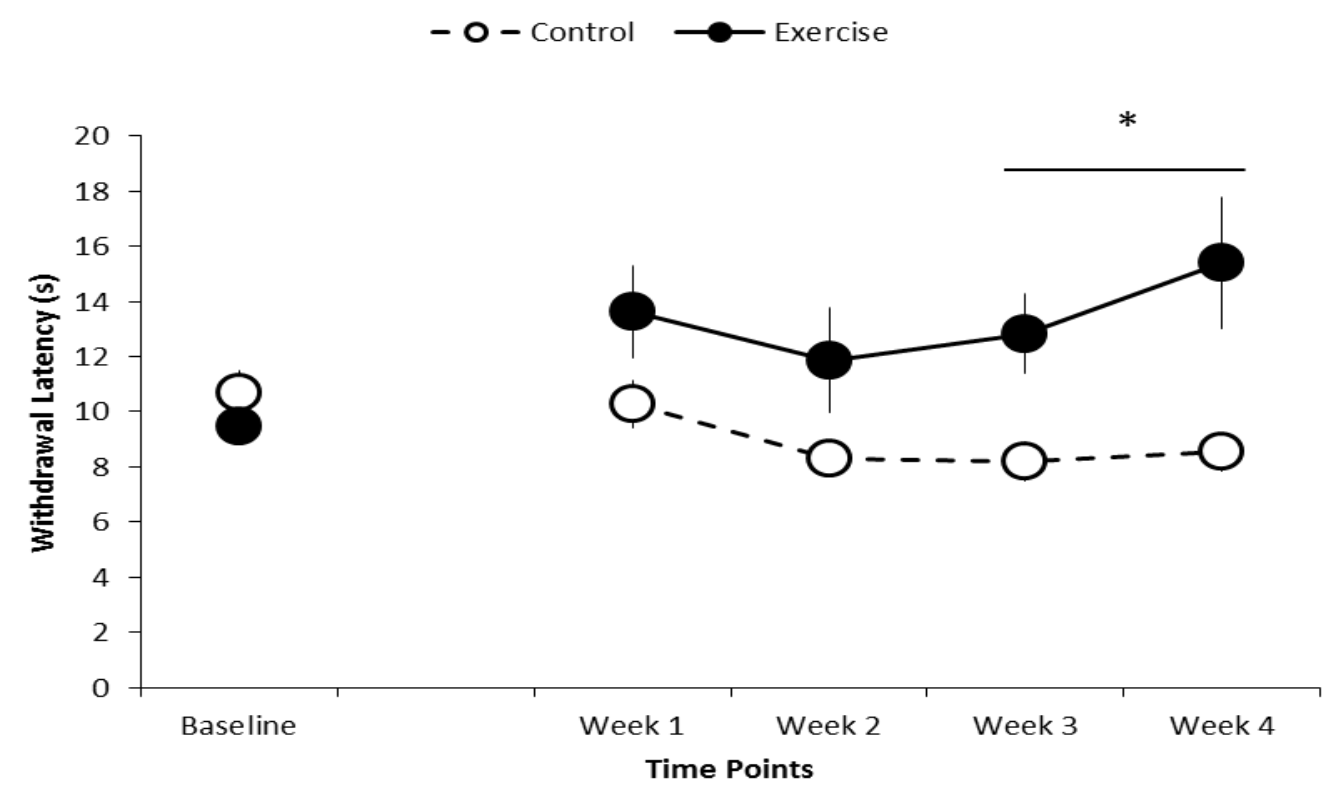

Figure 1. Voluntary, chronic exercise produces a hypoalgesic effect over 4 weeks. Mean (+/SEM) baseline thermal nociception of mice before and during 4 weeks of voluntary wheel running

${ }^{*} p \leq 0.01$ exercise vs. non-exercise at Week 3 and Week 4 time points

\subsection{Traumatic Stress and Chronic Exercise on Nociception}

To analyze the effects of chronic exercise and exposure to a predator odor stressor on thermal nociception, a $(2 \times 2 \times 2)$ mixed-factorial ANOVA was performed with the factors that include exercise (exercise vs. non-exercise), posttraumatic stress (non-stress vs. stress), and time (pre-stress baseline vs. $48 \mathrm{hr}$. post-stress) on thermal nociception. All scores were converted to a percentage of baseline due to variability in pre-stress scores from 4 weeks of voluntary exercise. Means and standard deviations are reported in Table 2. There was a significant main effect of exercise condition on thermal nociception $[F(1,20)=13.63,=p=0.001]$ with exercising mice $(M=107.00)$ demonstrating significantly higher scores on withdrawal latencies (decreased nociception) compared to non-exercising mice, $M=77.96$. There was a significant time $\mathrm{x}$ exercise condition interaction $[F(1,20)=13.63, p=0.001]$, indicating that withdrawal latencies for exercising mice differed compared to non-exercising mice over time as demonstrated in Figure 2. Post-hoc analyses using T-tests with Bonferroni corrections indicated that the exercise animals displayed significantly higher thermal withdrawal latencies at the post-stress time point when compared to non-exercising animals $(p=0.007)$. There was also significant interaction for time $\mathrm{x}$ exercise condition $\mathrm{x}$ stress condition $[F(1$, $20)=8.86, p=0.007]$. Post-hoc analyses for the time $\mathrm{x}$ exercise condition $\mathrm{x}$ stress condition interaction utilized T-tests with Bonferroni corrections and reflected significantly higher scores on withdrawal latencies in exercising, stressed animals than non-exercising, neutral mice $(p=0.01)$, non-exercising, stressed mice $(p=0.001)$, and exercising, neutral $(p=0.05)$ 
at the post-stress timeline. No other reported main effects or interactions were statistically significant. Data are summarized in Figure 2.

Table 2. The means and standard deviations for stress condition $\mathrm{x}$ exercise condition on withdrawal latencies at baseline and after stress exposure

\begin{tabular}{lccccccc}
\hline & \multicolumn{3}{c}{ Prestress } & & \multicolumn{3}{c}{$\underline{\text { Poststress }}$} \\
Withdrawal latencies & $M$ & $S D$ & $n$ & $M$ & $S D$ & $n$ \\
\hline Non-exercising neutral & 100 & 0 & 6 & 84.83 & 14.71 & 6 \\
Non-exercising stress & 100 & 0 & 6 & 71.10 & 19.53 & 6 \\
Exercising neutral & 100 & 0 & 6 & 90.45 & 16.90 & 6 \\
Exercising stress & 100 & 0 & 6 & 123.57 & 24.55 & 6 \\
\hline
\end{tabular}

Note. Neutral indicates mice were exposed to ambient air whereas stress indicates mice were exposed to predator odor stressor.

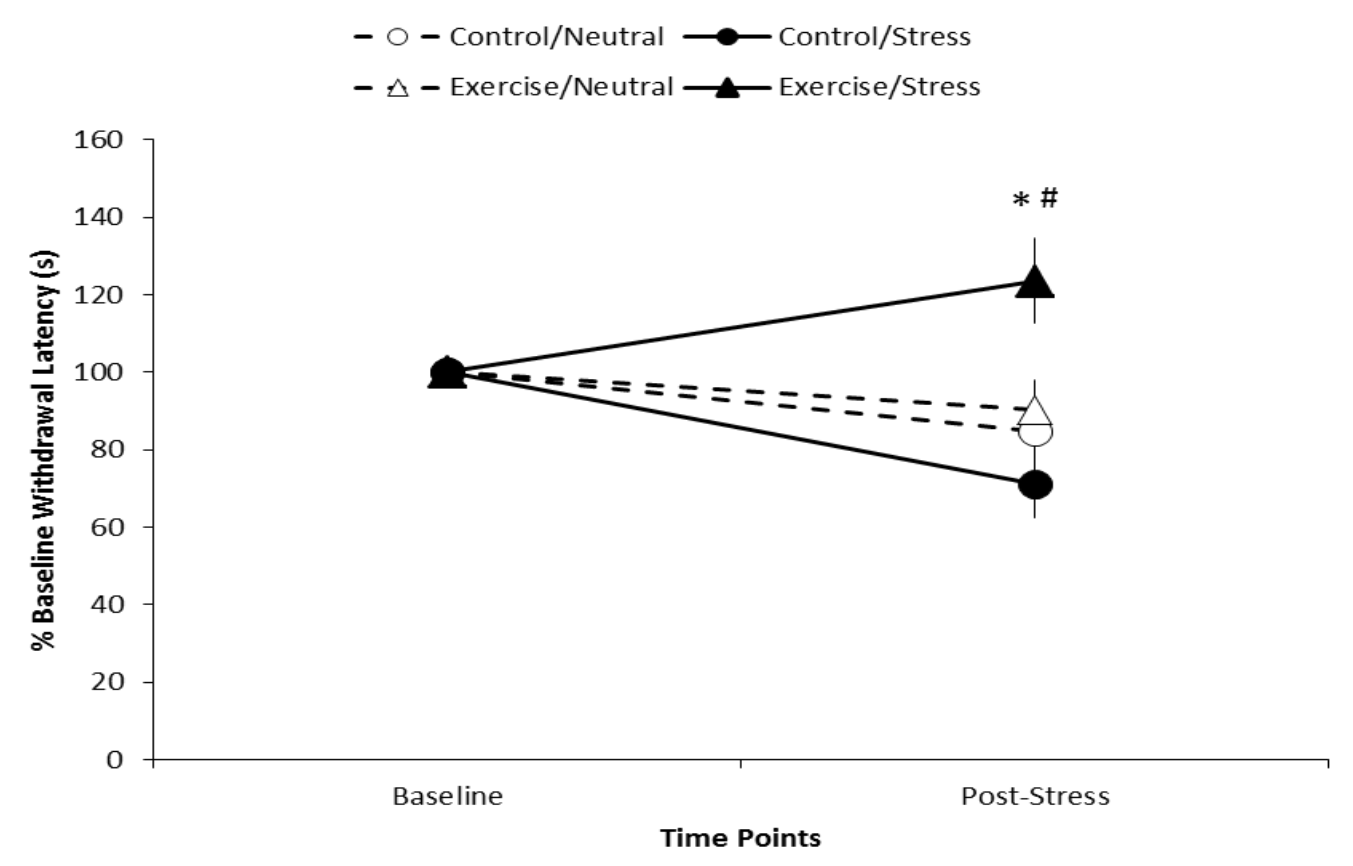

Figure 2. Voluntary, chronic exercise produces a hypoalgesic effect before and after a traumatic stressor. Mean (+/- SEM) pre-stress and post-stress (48 hours) thermal nociception scores of mice. Data were converted to a percentage of Week 4 pre-stress scores

$* p \leq 0.01$ pre-stress vs. post-stress for exercise/stress condition. $\# p \leq 0.01$ exercise/stress vs. exercise/neutral, no exercise/stress, and no exercise/neutral 


\section{Discussion}

The findings from this study supported the first hypothesis that chronic, voluntary exercise would decrease thermal nociception (hypoalgesia) in mice when compared to non-exercising controls. This was demonstrated by higher withdrawal latencies in exercising animals when compared to non-exercising mice over a 4-week period. More importantly, the hypoalgesic effect of chronic exercise increased over time as indicated by an interaction between time and condition that displayed larger differences between the groups over longer periods of exercise. In addition, the results provided partial support for the second hypothesis that stressed, non-exercising mice would demonstrate significantly higher nociception (pain-sensitivity or hyperalgesia) compared to their baseline nociceptive levels, stressed exercising mice, non-stressed exercising mice, and non-stressed no exercise controls. Following either predator odor or neutral odor, non-exercising animals displayed significantly lower nociceptive scores compared to stressed, exercising mice and exercising controls but did not statistically differ from non-exercising, non-stressed animals. This finding indicates a lack of hyperalgesic responses to a traumatic stressor in the mouse model due to no significant changes in nociception following the traumatic stressor in non-exercising animals. Interestingly, animals participating in voluntary exercise over a 4-week period show an additional hypoalgesic effect following the traumatic stressor as evidenced by an increase in withdrawal latencies following the traumatic stressor. All other conditions demonstrated a slight, non-significant decrease in withdrawal latencies after the odor exposure. This finding provides partial support for chronic exercise as a way to alleviate stress-induced hyperalgesia and has important implications for the treatment of symptoms following a traumatic stressor. These findings were consistent with current literature that supports exercise as an antinociceptive tool but was novel in examining the impact of chronic exercise on nociception in an animal stress model (Geisser et al., 2008).

\subsection{Chronic Exercise Decreases Nociception}

Crombie and other colleagues (2018) conducted related research examining the role of aerobic exercise on nociception and its impacts on the endocannabinoid (eCB) system and mood improvement in human participants. Although Crombie and other colleagues (2018) found that aerobic exercise elicited release of eCBs (indicative of pain relief) and improved mood in participants, the present study examined the impacts of acute bouts of exercise rather than chronic exercise regimens. To date, the majority of investigations have examined the role of exercise on nociception in short-term (1 bout of exercise) exercise models (Naugle et al., 2012; Kotlyn, Brellenthin, Cook, Sehgal \& Hillard, 2015). The current findings yield support for the effect of chronic exercise (exercise training) on reductions of nociception over time, impacting long-term pain processing. Geisser and other researchers (2008) also examined how increased physical fitness in rats plays a significant role in pain perception and demonstrates the benefits of chronic exercise. In their study, rats bred for higher aerobic capacity (HCR) demonstrated significantly higher pain thresholds compared to the animals bred with low aerobic capacity (LCR). Following exercise, HCR animals' pain thresholds returned to baseline faster than the LCR rats following exercise. Moreover, Hides and colleagues (2001) have also found that long-term specific exercise therapy in addition to 
medication and continuing normal daily functioning (e.g. going to work) has demonstrated to be more effective in the treatment of chronic pain than medical management and normal activity alone. Thus, findings from the current study along with additional research demonstrate how exercise should be incorporated long-term to be most effective in pain reduction (Geisser et al., 2008; Hides, Jull \& Richardson, 2001).

\subsection{Mechanisms for EIH}

Despite the growing body of evidence supporting the incorporation of long-term exercise into treatment programs for chronic pain conditions due to its ability to reduce pain sensitivity in humans and animals, the mechanisms behind the hypoalgesic effects are still being identified. A significant amount of research has attributed these effects to the opioid and endocannabinoid systems (eCB) in the brain (Crombie et al., 2018; Geisser et al., 2008) eCB signaling in the brain through $\mathrm{CB} 1$ receptors and the periphery has been specifically linked to the therapeutic benefits of exercise such as the regulation of nociception and stress as well as memory and emotional regulation (Gorzalka, Hill \& Hillard, 2008). Additionally, Kotlyn and colleagues (2015) demonstrated that following three sessions of isometric exercise, there was evidence of circulating concentrations of endocannabinoids and its related lipids. These results also demonstrated significantly increased pain thresholds following exercise in both opioid antagonist and control conditions providing support for a non-opioid mechanism.

Another possible explanation of the link between pain relief and chronic exercise may be linked to changes in CRF. Previous findings suggest that short-term exercise may be perceived as a controllable threat and elicit acute fluctuations in CRF, but chronic exercise has demonstrated to lower ACTH responses to CRF in animal models (Whitaker et al., 2016). Moreover, decreased levels of endogenous CRF have been associated with hypoalgesic effects as demonstrated by Yarushkina and other colleagues (2016). Although the current evidence did not directly examine ACTH responses to CRF, the impact of CRF on exercise-induced hypoalgesia should be considered for future studies due to the large role of CRF in stress related situations.

\subsection{Traumatic Stress and Hyperalgesia}

Exposure to repeated stress has produced alterations to hormonal release and nociception, particularly leading to post-stress hyperalgesia in animals and humans (Ibironke \& Mordi, 2018; Suarez-Roca et al., 2014). The findings from the current study indicate increased nociception following exposure to a stressor but were not statistically significant. These results may be explained by the phenomenon that humans can be exposed to a traumatic experience, but not all those individuals develop traumatic symptoms indicative of PTSD. In the United States, $70 \%$ of citizens have been previously exposed to a traumatic experience, but only $20 \%$ of those individuals go on to or currently struggle with PTSD (PTSD United, 2013). Evidently, stress exposure may not lead to behavioral and physiological changes in all animals or humans. However, current literature yields support for increased nociception following stress exposure in animals and humans (Itoga et al., 2016; Roltsche et al., 2014). Although forced exercise has shown to be perceived as a traumatic experience in rats and elicit hyperalgesia, the results from our study suggest that chronic voluntary exercise may 
serve as a buffer against the hyperalgesia effects following stress exposure. Crombie and other researchers (2018) have demonstrated support for the activation of endocannabinoid system in adults with and without PTSD following 30 minutes of moderate-intensity aerobic exercise. Furthermore, evidence indicates alterations in CRF levels and CRF stimulatory effects following exercise which is of great importance given the significant role of CRF in trauma-induced nociceptive changes.

\section{Limitations}

A limitation in our findings was that exposure to predator odor stressor did not have a significant effect on nociception. However, raw scores did indicate a hyperalgesic effect in about half of the animals that were exposed to the stressor. Therefore, the hyperalgesic stress effect was neutralized by those mice that were non-responsive to the stressor. In addition, $\mathrm{CRF}$ and $\mathrm{ACTH}$ levels were not measured as a variable and therefore, our current findings did not directly demonstrate the changes in $\mathrm{CRF}$ and its effects following chronic exercise and posttraumatic stress exposure.

\section{Conclusions}

Findings from the current study demonstrate that chronic, voluntary exercise decreases thermal nociception in mice over time as demonstrated by higher withdrawal latencies in exercising animals compared to non-exercising animals. Additionally, the results also indicate that chronic, voluntary exercise serves to alleviate stressed-induced hyperalgesia in mice following exposure to predator odor stressor. Stressed, non-exercising animals demonstrated the lowest scores on withdrawal latencies, indicating the physiological impacts that traumatic stress has on nociception. Moreover, these findings yield support for the utility of voluntary, long-term exercise programs in the treatment for those individuals who experience traumatic stressors. Future directions for research should target CRF and ACTH expression in methodology and protocol to more clearly understand its relationship to chronic exercise and exposure to stressors. Pharmaceutical inventions targeting CRF and ACTH expression may also be appropriate for future researchers to consider in the treatment of PTSD and for directions in future research.

\section{References}

Adamec, R., Fougere, D., \& Risbrough, V. (2010). CRF receptor blockade prevents initiation and consolidation of stress effects on affect in the predator stress model of PTSD. International Journal of Neuropsychopharmacology, 13(6), 747-757. https://doi.org/10.1017/S1461145709990496

American Psychiatric Association. (2013). Diagnostical statistical manual of mental disorders, 5th edn. Washington D.C. https://doi.org/10.1176/appi.books.9780890425596

Asmundson, G. J. G., \& Katz, J. (2008). Understanding pain and posttraumatic stress disorder comorbidity: Do pathological responses to trauma alter perception of pain? Pain, 138, 247-249. https://doi.org/10.1016/j.pain.2008.06.020 
Asmundson, G. J. G., Coons, M. J., Taylor, S., \& Katz, J. (2002). PTSD and the experience of pain: Research and clinical implications of shared vulnerability and mutual maintenance models. Canadian Journal of Psychiatry, 47, 930-937.

https://doi.org/10.1177/070674370204701004

Baiamonte, B. A., Kraemer, R. R., Chabreck, C. N., Reynolds, M. L., McCaleb, K. M., Shaneen, G. L., \& Hollander, D. B. (2016). Exercise-induced hypoalgesia: Pain tolerance, preference and tolerance for exercise intensity, and physiological correlates following dynamic circuit resistance exercise. Journal of Sports Sciences, 35(18), 1-7.

https://doi.org/10.1080/02640414.2016.1239833

Crombie, K. M., Brellenthin, A. G., Hillard, C. J., \& Kotlyn, K. F. (2018). Psychobiological responses to aerobic exercise in individuals with posttraumatic stress disorder. Journal of Traumatic Stress, 1-12. https://doi.org/10.1002/jts.22253

Defrin, R., Ginzburg, K., Solomon, Z., Polad, E., Bloch, M., Govezensky, M., \& Schreiber, S. (2008). Quantitative testing of pain perception in subjects with PTSD - Implications for the mechanism of coexistence between PTSD and chronic pain. Pain, 138(2), 450-459. https://doi.org/10.1016/j.pain.2008.05.006

Droste, S. K., Chandramohan, Y., Hill, L. E., Linthorst, A. C. E., \& Reul, J. M. H. M. (2007). Voluntary exercise impacts on the hypothalamic-pituitary-adrenocortical axis mainly at the adrenal level. Neuroendocrinology, 86, 26-37. https://doi.org/10.1159/000104770

Duclos, M., Corcuff J. B., Rashedi, M, Fougere, V., \& Manier, G. (1997). Trainer versus untrained men: Different immediate post-exercise repsonses of the pituitary adrenal axis. A preliminary study. Eur J Appl Physiol Occup Physiol., 75(4), 343-350.

https://doi.org/10.1007/s004210050170

Duclos, M., Guoarne, C., \& Bonnemaison, D. (2016). Acute and chronic effects of exercise on tissue sensitivity to glucocorticoids. Journal of Applied Physiology, 94(3).

https://doi.org/10.1152/japplphysiol.00108.2002

Edwards, S., Vendruscolo, L. F., Schlosburg, J. E., Misra, K. K., Wee, S., Park, P. E., ... Kooch, G. F. (2012). Development of mechanical hypersensitivity in rats during heroin and ethanol dependence: Alleviation by $\mathrm{CRF}_{1}$ receptor antagonism. Neuropharmacology, 62, 1142-1151. https://doi.org/10.1016/j.neuropharm.2011.11.006

Fetzner, M. G., \& Asmundson, G. J. G. (2015). Aerobic exercise reduces symptoms of posttraumatic stress disorder: A randomized controlled trial. Cognitive Behavior Therapy, 44(4), 301-313. https://doi.org/10.1080/16506073.2014.916745

Geisser, M. E., Wang, W., Smuck. M., Koch, L. G., Britton, S. L., \& Lydic, R. (2008). Nociception before and after exercise in rats bred for high and low aerobic capacity. Neuroscience Letters, 443(1), 37-40. https://doi.org/10.1016/j.neulet.2008.07.040 


\section{$\Lambda$ Macrothink}

Journal of Biology and Life Science ISSN 2157-6076

Geneen, L. J., Moore, R. A., Clarke, C., Martin, D., Colvin, L. A., \& Smith, B. H. (2001). Physical activity and exercise for chronic pain in adults: an overview of Cochrane Reviews. Cochrane Database System Review, 4.

Gorzalka, B. B., Hill, M. N., \& Hillard, C. J. (2008). Regulation of endocannabinoid signaling by stress: Implications for stress-related affective disorders. Neuroscience Biobehavioral Reviews, 32, 1152-1160. https://doi.org/10.1016/j.neubiorev.2008.03.004

Gouarne, C., Groussard, C., Gratas-Delamarche, D. P., \& Duclos, M. (2005). Overnight urinary cortisol and cortisone add new insights into adaption into training. Med. Sci. Sports Exerc., 37(7), 1157-1167. https://doi.org/10.1249/01.mss.0000170099.10038.3b

Hides, J., Jull, G. A., \& Richardson, C. A. (2001). Long-term effects of specific stabilizing exercise for first-episode low back pain. Exercise Physiology and Physical Exam., 26(11), e243-248. https://doi.org/10.1097/00007632-200106010-00004

Ibironke, G. F., \& Mordi, N. E. (2011). Effect of restraint stress on nociceptive responses in rats: the role of the histamergic system. Nigeria Journal of Physiological Science, 139-141. Available from http://www.bioline.org.br/pdf?np11021 on 23 July 2018

Itoga, C. A., Hellard, R. E. A., Whitaker, A. M., Lu, Y., Schreiber, A. L., Baynes, B. B., ... Gilpin, N. W. (2016). Traumatic stress promotes hyperalgesia via corticotropin-releasing factor-1 receptor (CRFI) signaling in central amygdala. Neuropsychopharmacology, 41, 2463-2472. https://doi.org/10.1038/npp.2016.44

Ji, G., \& Neugebauer, V. (2007). Differential effects of CRF1 and CRF2 receptor antagonists on pain-related sensitization of neurons in central nucleus of the amygdala. Journal of Neuropharmacology, 97, 3893-3904. https://doi.org/10.1152/jn.00135.2007

Ji, G., \& Neugebauer, V. (2008). Pro- and anti-nociceptive effects of corticotropin-releasing factor (CRF) in central amygdala neurons are mediated through different receptors. Journal of Neuropharmacology, 25, 289-297.

Kotlyn, K. F. (2000). Analgesia following exercise. Journal of Sports Medicine, 2, 85-98. https://doi.org/10.2165/00007256-200029020-00002

Kotlyn, K. F., \& Arbogast, R. W. (1998). Perception of pain after resistance exercise. British Journal of Sports Medicine, 32, 20-24. https://doi.org/10.1136/bjsm.32.1.20

Kotlyn, K. F., Brellenthin, A. G., Cook, D. B., Sehgal, N., \& Hillard, C. J. (2015). Mechanisms of exercise-induced hypoalgesia. Journal of Pain., 15(12), 1294-1304. https://doi.org/10.1016/j.jpain.2014.09.006

Mostoufi, S., Godfrey, K. M., Ahumada, S. M., Hossain, N., Song, T., Wright, L. J., ... Afair, N. (2014). Pain sensitivity in posttraumatic stress disorder and other anxiety disorders: A preliminary case control study. Annals of General Psychiatry, 13, 31. https://doi.org/10.1186/s12991-014-0031-1 
Naugle, K M., Fillingim, R. B., \& Riley, III, J. L. (2012). A meta-analytic review of the hypoalgesic effects of exercise. The Journal of Pain., 13(12), 1139-1150. https://doi.org/10.1016/j.jpain.2012.09.006

Powers, M. B., Medina, J. L., Burns, S., Kauffman, B. Y., Monfils, M., Asmundson, G J. G., ... Smits, J. A. J. (2015). Exercise augmentation of exposure therapy for PTSD: rationale and pilot efficacy data. Cognitive Behavior Therapy, 44(4), 314-327. https://doi.org/10.1080/16506073.2015.1012740

PTSD United (2013). PTSD statistics. Available on October 17, 2017 from http://www.ptsdunited.org/ptsd-statistics-2/

Roltsch, E. A., Baynes, B. B., Mayeaux, J. P., Whitaker, A. M., Baiamonte, B. A., \& Gilpin, N. W. (2014). Predator odor stress alters corticotropin-releasing factor-1 receptor (CRF1R)-dependent behaviors in rats. Neuropharmacology, 79, 83-89. https://doi.org/10.1016/j.neuropharm.2013.11.005

Suarez-Roca, H., Quintero, L., Avila, R., Medina, S., De Freitas, M., \& Cardenas, R. (2014). Central immune overactivation in the presence of reduced plasma corticosterone contributes to swim stress-induced hyperalgesia. Brain Research Bulletin., 100, 61-69. https://doi.org/10.1016/j.brainresbull.2013.11.003

U.S. Department of Veteran Affairs. (2017). PTSD: National Center for PTSD. U.S. Department of Veteran Affairs (2017). Available on June 11, 2017 from https://www.ptsd.va.gov/professional/PTSD-overview/dsm5_criteria_ptsd.asp

Watanabe, T., Morimoto, A., Sakata, Y., Wada, M., \& Murakami, N. (1991). The effect of chronic exercise on the pituitary-adrenocortical response in conscious rats. $J$ Physiol., 439, 691-699. https://doi.org/10.1113/jphysiol.1991.sp018688

Whitaker, A. M., Farooq, M. A., Edwards, S., \& Gilpin, N. W. (2016). Post-traumatic stress avoidance is attenuated by corticosterone and associated with brain levels of steroid receptor co-activator-1 in rats. Stress, 19(1), 69-77. https://doi.org/10.3109/10253890.2015.1094689

Yarushkinda, N. I., Bagaeva, T. R., \& Filaretova, L. P. (2016). Effects of corticotropin-releasing factor $(\mathrm{CRF})$ on somatic pain sensitivity in conscious rats: Involvement of types 1 and 2 CRF receptors. Neuroscience and Behavioral Physiology; 46(4), 472-477. https://doi.org/10.1007/s11055-016-0260-7

\section{Copyright Disclaimer}

Copyright for this article is retained by the author(s), with first publication rights granted to the journal.

This is an open-access article distributed under the terms and conditions of the Creative Commons Attribution license (http://creativecommons.org/licenses/by/3.0/). 